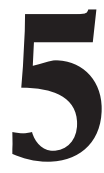

\title{
CALIDAD DE VIDA Y COMPETENCIAS DE EMPLEABILIDAD EN PERSONAS TRABAJADORAS EN CENTROS ESPECIALES DE EMPLEO
}

\author{
(QUALITY OF LIFE AND EMPLOYABILITY SKILLS IN WORKERS IN \\ SPECIAL EMPLOYMENT CENTERS)
}

Cristina Laborda Molla

Mercè Jariot García

Henar González Fernández

Universitat Autònoma de Barcelona

DOI: 10.5944/educXX1.26570

\section{Cómo referenciar este artículo/How to reference this article:}

Laborda Molla, C.; Jariot García, M. y González Fernández, H. (2021). Calidad de vida y competencias de empleabilidad en personas trabajadoras en centros especiales de empleo. Educación XX1, 24(1), 117-139, https://doi.org/10.5944/educXX1.26570

Laborda Molla, C.; Jariot García, M. \& González Fernández, H. (2021). Quality of life and employability skills in workers in special employment centers. Educación XX1, 24(1), 117-139, https://doi.org/10.5944/educXX1.26570

\section{RESUMEN}

Este artículo profundiza en la relación entre competencias de empleabilidad (CE) y calidad de vida (CV) en personas con discapacidad intelectual leve y moderada que trabajan en un centro especial de empleo (CEE), como elemento determinante en su nivel de inclusión sociocomunitaria. La información se recogió mediante un cuestionario de competencias de empleabilidad de elaboración propia, así como a través de la escala de calidad de vida de Schalock y Verdugo (2008), que se pasó a 100 trabajadores de 15 CEE. Las personas de la muestra presentan un bajo nivel de calidad de vida, pero alto en competencias de empleabilidad. No existe relación entre ambas variables. En cuanto a la calidad de vida, los jóvenes muestran mejores niveles de desarrollo personal que los mayores 
de 45 años y los hombres presentan mejores resultados en las relaciones interpersonales. Los altos niveles competenciales no difieren en función de la edad ni del género. Los resultados muestran la necesidad de una acción educativa longitudinal centrada en la persona, iniciada ya en la etapa de escolarización, para promover los dominios de la calidad de vida y que esta continúe a lo largo de la vida. Por otro lado, la formación laboral desarrollada desde los CEE evidencia buenos resultados en las competencias de las personas participantes en el estudio. Los colectivos específicos que requieren acciones prioritarias son el de personas mayores de 45 años, para promover un envejecimiento activo de calidad, así como el de mujeres, para evitar la doble discriminación a la que se ven sometidas (discapacidad y género).

\section{PALABRAS CLAVE}

Calidad de vida, competencias para la vida, discapacidad intelectual, empleo, empleabilidad

\section{ABSTRACT}

This article goes in depth in the relationship between employability skills and quality of life of people with mild and moderate intellectual disability who work in a special employment center (CEE), as a determining factor in their level of socio-community inclusion. Data was collected by means of a questionnaire on labor competences (own elaboration), as well as through the scale of quality of life by Schalock and Verdugo (2008) with 100 workers of $15 \mathrm{CEE}$. The worker's supervisor answered both. People in the sample have a low level of quality of life, but high in job skills. There is no relationship between both variables. In terms of quality of life, young people show better levels of personal development than those over 45 years of age; and men present better results in interpersonal relationships. High levels of competence do not differ according to age or gender. Results show the need for a longitudinal educational action centered on the person, already initiated in schooling, to promote the domains of the quality of life and to continue throughout life. On the other hand, job training developed in the CEE shows good results in the professional skills of participants in the study. The specific groups that require priority actions are those over 45 years old, to promote quality active aging, as well as women, to avoid double discrimination to which they are subjected (disability and gender). 


\section{KEYWORDS}

Quality of life, life skills, intellectual disability, employment, employability

\section{INTRODUCCIÓN}

El trabajo es un factor determinante para la realización personal de las personas con discapacidad intetelectual (DI), aportándoles seguridad personal (Mafla et al., 2019); convirtiéndose en una herramienta imprescindible para su inclusión socio-comunitaria y condición necesaria para conseguir el equilibrio entre el "ser individual" y el "ser social" (Pegalajar y Xandri, 2015). El colectivo de personas con DI muestra dificultades específicas en la obtención de un empleo (Riaño-Galán et al., 2016), por lo que se hace necesaria la existencia de soportes que compensen estas dificultades, aflorando el principio de equidad que garantice una verdadera inclusión sociolaboral.

Las características de la economía y del mercado laboral influyen negativamente en la inclusión, y esta inequidad, tanto laboral como económica, incide de especial manera en colectivos vulnerados y excluidos, como es el de personas con discapacidad, y concretamente con DI.

De hecho, incluso con un trabajo, las personas con DI se ven excluidas del empleo ordinario, debido a barreras estructurales (políticas de empleo, provisión de ayudas y recursos, prejuicios, bajas expectativas, etc.) que les limitan la inclusión sociolaboral (Grup de Treball d’Orientació, 2010).

Para minimizar los efectos de la exclusión, y siguiendo los parámetros de De la Fuente y González-Castro (2009) es necesario contar con redes de apoyo, con políticas específicas, con oportunidades para la participación, y con estabilidad laboral. Se considera la inserción laboral como una potente herramienta de inclusión socio-comunitaria, indicador indispensable de la calidad de vida (CV) de las personas y factor determinante en las relaciones que la persona establece con su entorno (Pegalajar, y Xandri, 2015). Para poder conseguir la inclusión socio-comunitaria, se debe primero asegurar la inserción laboral y, después, el desarrollo profesional de las personas con DI, ambos elementos indispensables para avanzar hacia la inserción comunitaria.

Este artículo se centra en el desarrollo de las competencias de empleabilidad y la calidad de vida de personas con DI en entornos protegidos, concretamente en centros especiales de empleo (CEE) y la CV. 


\section{COMPETENCIAS DE EMPLEABILIDAD Y CALIDAD DE VIDA}

El desarrollo de competencias de empleabilidad en entornos laborales es clave para el desempeño laboral satisfactorio de personas con DI. Gilson et al. (2017) relacionan las competencias de empleabilidad con la aplicación del conocimiento general a contextos profesionales específicos para la obtención de resultados esperados, según los estándares productivos de un puesto de trabajo determinado. Por tanto, se trata de la conexión de los parámetros individuales y sociales de la persona trabajadora, que aporta su itinerario vital en cada caso, más que algo constitutivo del puesto de trabajo (Docampo y Morán, 2014).

Blanco (2008) apunta como competencias de empleabilidad el conocimiento integrado, las habilidades, las actitudes y los valores relacionados con un contexto específico, que permiten a las personas responder a problemáticas concretas. No obstante, estas competencias tienen otros efectos a parte de su impacto directo en el éxito laboral, puesto que se relacionan directamente con la CV de las personas. La CV es definida por Schalock et al. (2010) como un fenómeno multidimensional, que se estructura a partir de 8 dominios divididos en 3 áreas: independencia (desarrollo personal, autodeterminación y relaciones interpersonales); participación social (inclusión social, derechos y bienestar emocional); bienestar (material y físico). Estos dominios son comunes para todas las personas, aunque varian en su relevancia, pues se ven influenciados por características tanto personales como ambientales.

La relación entre las competencias de empleabilidad y la CV parte de la consideración que el desempeño laboral contribuye al desarrollo personal. Según autores como Jahoda et al. (2008) creando rutinas diarias, proporcionando autonomía económica, incorporando a la persona a nuevos contextos (distintos del familiar), y predisponiéndola a nuevas circunstancias favorecedoras de su crecimiento personal. Otros estudiosos destacan que el trabajo ayuda al desarrollo de las competencias sociales al ubicar a la persona en un entorno laboral determinado en el que interacciona y establece nuevas relaciones (Fillary y Pernice, 2005). Finalmente, Stephens et al. (2005) destacan las habilidades cognitivas como el ámbito principal de mejora promovida desde el desempeño laboral, puesto que la persona está expuesta a situaciones competitivas. A nivel general, a mayor desarrollo de las competencias de empleabilidad de la persona, mayor nivel de éxito en el puesto de trabajo (Holmes \& Fillary, 2000).

Tener un trabajo es crucial para el desarrollo individual de una persona adulta con DI (Ellenkamp et al., 2016; Lindstrom et al., 2011; Nota et al., 2010). Conseguir un empleo es un logro, y más cuando es el primer paso 
hacia la materialización de la vida independiente, un símbolo de ciudadanía y de participación comunitaria.

González y Laborda (2017) identifican una concordancia entre el nivel de DI, la CV y las competencias de empleabilidad en entornos laborales de este colectivo. Roselló y Verger (2008) establecen una correlación entre el grado de DI, el nivel de desarrollo personal y las posibilidades de conseguir un puesto de trabajo. En este sentido parece obvio que el conocimiento del nivel competencial sea un elemento clave para el éxito de la inserción profesional. Consecuentemente, el diseño de instrumentos adaptados para la evaluación de competencias abre caminos para que las personas con DI puedan ser evaluadas por lo que realmente "saben hacer", lo que permitirá superar las dificultades reiteradas a la hora de demostrar sus habilidades, por la inadecuación de los métodos utilizados (Docampo y Morán, 2014).

\section{CENTROS ESPECIALES DE EMPLEO Y DESARROLLO LABORAL}

La normativa aplicable a los CEE se rige por el Real Decreto de 2275/1985 de 4 de diciembre. Desde entonces, no ha habido modificaciones sustanciales en su organización.

Son empresas que proporcionan un puesto de trabajo remunerado a personas con discapacidad. Siguiendo parámetros sociales y laborales y atendiendo a criterios de tipología de centro, grado de apoyo y tipo de tarea (Laborda y González, 2017a), los CEE se pueden considerar: 1) específicos: especialmente diseñados para personas con discapacidad; 2) parciales: el tipo y grado de apoyo no es ni completo ni puntual, y se refiere a aspectos concretos del quehacer profesional; 3) productivos: su objetivo es de provecho empresarial.

Estas características implican que se englobe a los CEE dentro de la categoría de trabajo protegido y que, aunque su objetivo sea netamente productivo, desarrollen también una función social, velando por el ajuste personal y social de los trabajadores y desplegando un abanico de prestación de servicios (soporte psicológico, oferta de ocio, entre otros). Su plantilla debe estar compuesta en un mínimo del 70\% por trabajadores con certificado de discapacidad (igual o superior al 33\%). Por último, otra característica determinante del CEE es que debería suponer un elemento de tránsito hacia fórmulas laborales más inclusivas (como la del trabajo con apoyo), aunque en vez de convertirse en puente hacia el trabajo ordinario, se ha convertido en una modalidad segregadora, puesto que para la mayoría de sus trabajadores se convierte en su último destino laboral (Calderón, y Calderón, 2012; Moratalla, 2016; Rodríguez, 2019). 
Parece lógico suponer que la reubicación y posibilidad de avance hacia enfoques profesionales más normalizados y hacia una mayor inclusión socio-comunitaria, dependa significativamente del grado de desarrollo de las propias competencias de empleabilidad, aunque a su vez, la propia tipología de centro laboral sea un elemento contributivo al desenvolvimiento de dichas competencias. Sin embargo, el estudio de Romero et al. (2017) evidencia la dificultad de desarrollar las competencias profesionales y la carrera de este colectivo.

Por otro lado, la configuración del mundo laboral en la sociedad actual dificulta la normalización laboral y aboca a este colectivo a integrarse en un CEE, aunque de no ser por las dificultades ya detectadas en los procesos formativos (Laborda y González, 2017b) su capacidad de trabajo los abocaría a inserciones laborales más normalizadas.

\section{MÉTODO}

El objetivo general de esta investigación es comprobar si el desarrollo de competencias de empleabilidad en entornos laborales influye en la CV de los trabajadores de los CEE, y a la inversa. Como objetivos específicos, se pretende indagar si el género o la edad son determinantes en dicha relación.

Se optó por una metodología cuantitativa no experimental observando el fenómeno en su contexto natural. Se desarrolló un estudio por encuesta descriptivo y comparativo-causal.

El diseño de la investigación contempló los permisos y las recomendaciones éticas, puesto que el tratamiento de los datos fue siempre anónimo y se contó con la aprobación de los participantes.

\section{Participantes}

Ante la imposibilidad de realizar un muestreo aleatorio efectivo se optó por un muestreo no probabilístico de tipo incidental. Se solicitó la participación voluntaria de los supervisores de los trabajadores con DI de los 15 CEE a través de un cuestionario presencial. El criterio de elección correspondió a la distribución de centros públicos/privados de la provincia de Barcelona y a la posterior voluntariedad de los mismos. Así, el 9\% de los centros de la muestra eran de carácter público, y el 91\% privado. Estos datos se asemejan a la distribución de la población de CEE de la provincia de Barcelona: públicos 10\% (19) y privados 90\% (166). 
Con el objetivo de controlar la influencia de las variables experiencia profesional, nivel de comunicación y grado de discapacidad, se determinó como condición de participación que los trabajadores con DI llevaran más de 1 año realizando la misma tarea, tuvieran buen nivel comunicativo y presentaran un nivel de DI leve o moderada.

Los supervisores de los trabajadores se encargaron de evaluarlos. Para obtener información válida sobre los trabajadores a evaluar, se les requirió una antigüedad en el centro de 1 año o superior, y de 6 meses como supervisores de los trabajadores a los que debían evaluar, puesto que era importante un buen conocimiento de la persona.

El número de participantes fue de 100 trabajadores con DI de distintos CEE, con una media de edad de 42.6 años, distribuida de la siguiente manera: $18.1 \%$ entre $21-34$ años ; $41.5 \%$ entre $35-45$ años y $40.4 \%$ entre 46-65 años. 51 fueron hombres y 49 mujeres; el 59\% con un grado de DI leve y el $41 \%$ moderado. El 29\% eran hombres con un grado de DI leve, el 22\% hombres con uno moderado, el 30\% mujeres con un grado de DI leve y el $19 \%$ mujeres con DI moderado.

\section{Instrumentos}

Para la dimensión competencias de empleabilidad se elaboró un cuestionario adhoc, basado en los trabajos de Lucas et al., (2005) y Docampo y Morán (2014), clasificadas en 4 tipologias: cognitivas y de aprendizaje, funcionales, sociales-actitudinales y comunicativas.

El primer bloque recogía información sobre variables sociodemográficas: género, edad, grado de DI, centro de trabajo y tamaño de este. El segundo, incluía preguntas sobre los 25 elementos de competencia de las cuatro tipologías, que se presentan en la Tabla 1. Todas las preguntas debían ser fácilmente observables por el supervisor, que debía puntuarlos en una escala del 1 al 4, de menor a mayor desarrollo. 
Tabla 1

Bloques y competencias incluidas en el cuestionario

ESCALA

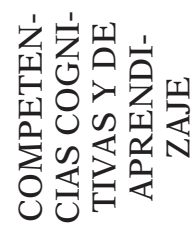

Atención selectiva

$\begin{array}{llll}1 & 2 & 3 & 4\end{array}$

Atención sostenida

$\begin{array}{llll}1 & 2 & 3 & 4\end{array}$

Detección de errores

$\begin{array}{llll}1 & 2 & 3 & 4\end{array}$

Potencial de aprendizaje

$\begin{array}{llll}1 & 2 & 3 & 4\end{array}$

Capacidad para sistematizar las tareas

$\begin{array}{llll}1 & 2 & 3 & 4\end{array}$

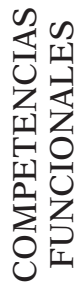

Planificación y organización

$\begin{array}{llll}1 & 2 & 3 & 4\end{array}$

Resistencia delante de las tareas repetitivas

$\begin{array}{llll}1 & 2 & 3 & 4\end{array}$

Resolución de problemas

$\begin{array}{llll}1 & 2 & 3 & 4\end{array}$

Capacidad para solicitar ayuda cuando lo necesite

$\begin{array}{llll}1 & 2 & 3 & 4\end{array}$

Habilidades manipulativas

$\begin{array}{llll}1 & 2 & 3 & 4\end{array}$

Relación con los profesionales

$\begin{array}{llll}1 & 2 & 3 & 4\end{array}$

Relación con los compañeros

$\begin{array}{llll}1 & 2 & 3 & 4\end{array}$

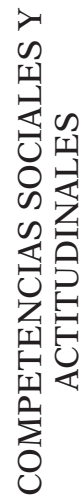

Capacidad de trabajo en equipo

$\begin{array}{llll}1 & 2 & 3 & 4\end{array}$

Respetar y cuidar los materiales / herramientas

$\begin{array}{llll}1 & 2 & 3 & 4\end{array}$

Nivel de responsabilidad

$\begin{array}{llll}1 & 2 & 3 & 4\end{array}$

Nivel de iniciativa

$\begin{array}{llll}1 & 2 & 3 & 4\end{array}$

Aceptación de les críticas

$\begin{array}{llll}1 & 2 & 3 & 4\end{array}$

Nivel de autonomía

$\begin{array}{llll}1 & 2 & 3 & 4\end{array}$

Aceptación de la autoridad

$\begin{array}{llll}1 & 2 & 3 & 4\end{array}$

Nivel de flexibilidad y de adaptación a los cambios

$\begin{array}{llll}1 & 2 & 3 & 4\end{array}$

Capacidad de motivación

$\begin{array}{llll}1 & 2 & 3 & 4\end{array}$

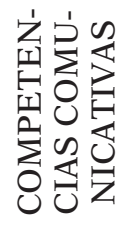

Comprensión de instrucciones verbales

$\begin{array}{llll}1 & 2 & 3 & 4\end{array}$

Comprensión de las instrucciones escritas

$\begin{array}{llll}1 & 2 & 3 & 4\end{array}$

Expresión oral

$\begin{array}{llll}1 & 2 & 3 & 4\end{array}$

Expresión escrita

$\begin{array}{llll}1 & 2 & 3 & 4\end{array}$

Para la dimensión CV, se utilizó la escala GENCAT de Schalock y Verdugo (Verdugo, Arias, Gómez. y Schalock, 2008), validado para personas 
con DI. Su consistencia interna es buena $(\alpha=.92)$, y tiene un bajo estándar de error de medida (SEM=6.92).

Se compone de 8 escalas, en correspondencia con los 8 dominios del modelo multidimensional de Schalock y Verdugo (2008). Se responde por un supervisor a través de una escala de frecuencia con 4 opciones (siendo 1 nunca o raras veces y 4 siempre o casi siempre), que aporta una puntuación para cada dimensión, y un índice global de CV.

Para el análisis de los datos de CV se emplearon las puntuaciones estándar del instrumento (media 10 y desviación típica 3), calculando el perfil a partir de las ocho dimensiones, y el índice a partir de las ocho subescalas. Se utilizó el baremo para personas con DI.

\section{Procedimiento y análisis de los datos}

El contacto inicial se estableció vía correo electrónico. A partir de su aceptación , se agendó un encuentro presencial presentándose los objetivos y la metodología del estudio. En una segunda reunión se planificó el proceso de recogida de datos y el de retorno de los resultados.

En el análisis de los datos se emplearon las puntuaciones estándar para establecer la posición relativa de los participantes dentro de su muestra de tipificación, así como los percentiles para poder indicar el porcentaje de personas que tienen una puntuación superior o inferior a la población de referencia a partir de los baremos del test.

Se calcularon las medias, desviaciones típicas y medianas de todos los ítems del cuestionario de competencias de empleabilidad y se efectuaron representaciones de distribuciones de frecuencias. Se realizó un análisis descriptivo de las puntuaciones estándar de la escala de CV. Posteriormente se analizaron las relaciones existentes entre el índice de y el nivel de competencia general, y las subescalas de CV y la edad ( $\mathrm{r}$ de Pearson). También se desarrolló un análisis de los datos mediante la prueba t-test para grupos independientes, para comprobar las diferencias en el desarrollo de las competencias y de los niveles de CV en función del género.

Posteriormente, y para comprobar si existían o no diferencias en cuanto al perfil de CV y al nivel competencial en función del género, se planteó un Análisis de Varianza (ANOVA) de un factor con tres niveles, en el que el factor estaba compuesto por la variable cualitativa intervalo de edad, con tres niveles (21-34 años, 35-45 años y 46-65 años); las variables de respuesta cuantitativas eran: el nivel general de competencias, el de 
las subescalas, el índice de CV y los perfiles de CV. Se realizó el contraste de Levene sobre la igualdad de las varianzas de error, para contrastar la hipótesis de que la varianza de error de la variable dependiente era igual a lo largo de todos los grupos y se procedió a la realización de comparaciones múltiples (pruebas post hoc) mediante la DHS de Tukey, para determinar en qué escalas concretas se producían diferencias.

\section{RESULTADOS}

\section{Índice de calidad de vida}

Los resultados globales muestran niveles diferenciados del índice de $\mathrm{CV}$, puesto que las puntuaciones estándar oscilan entre el 61 y el 102. La media de la puntuación estándar es de 76.6, la mediana de 76 y la moda 76.

Comparando la muestra con la población, encontramos que los sujetos se sitúan entre el percentil 1 y el 55. La puntuación media del percentil es de 8.5, lo que significa (tomando la interpretación de la prueba utilizada) que, en conjunto, los participantes tienen una puntuación superior solo al $8.5 \%$ de la población. La mediana se sitúa en el percentil 5, significando que solo el $5 \%$ de la muestra tiene una puntuación superior al de la población, y la moda se ubica en el percentil 5, observando que un 15\% de los participantes se sitúan en este percentil.

El análisis del perfil de CV, teniendo en cuenta las puntuaciones estándar de cada una de las ocho dimensiones o subescalas, quedó de la manera siguiente (Figura 1)

Figura 1

Perfil de CV de los participantes

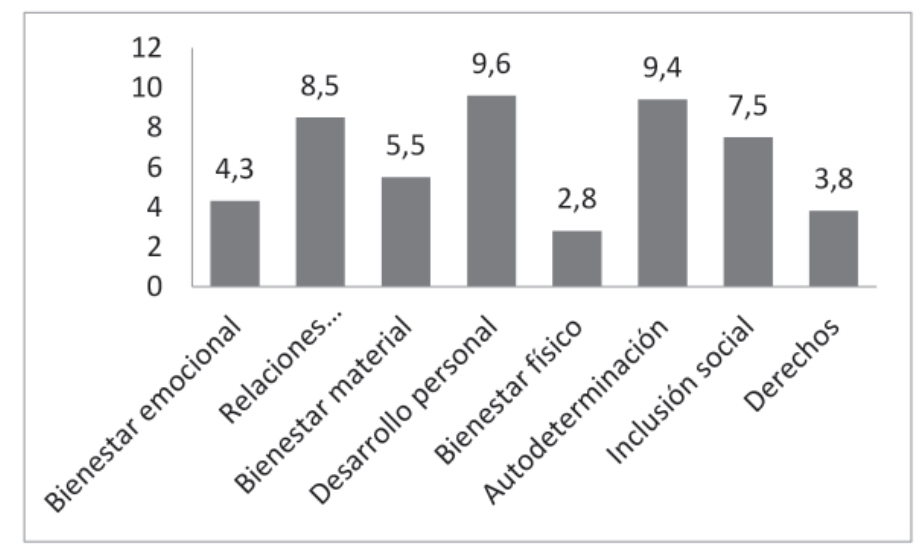


En la escala de bienestar emocional, media y mediana coinciden en 8 y la moda es 9. En la escala de relaciones interpersonales no existe dispersión, la mediana y la moda coinciden en la puntuación 8; lo mismo ocurre en la escala de bienestar emocional en 5. La escala de desarrollo personal media y mediana coinciden en 9.5, mientras que la moda es 8 . En la escala de bienestar físico la mediana es 2 y la moda 1. En la de autodeterminación coinciden mediana y moda en 10, en la de inclusión social en 8 . En la escala de derechos es donde se observa una mayor dispersión, con una mediana de 4 y una moda de 1 .

Al comparar el porcentaje de las puntuaciones de la muestra con la población de referencia para cada una de las escalas, podemos concluir que la muestra con la que trabajamos:

- Se sitúa por debajo del 72.7\% de las personas con DI en Bienestar Emocional.

- Se ubica por debajo del 65.9\% de la población con DI en Relaciones Interpersonales.

- Tiene una menor puntuación en Bienestar Material que el 84.8\%.

- Ha obtenido peores puntuaciones en Desarrollo Personal que el $55 \%$.

- Destaca en los bajos índices de Bienestar Físico, obteniendo puntuaciones inferiores al $97.8 \%$ de las personas con DI.

- Muestra un índice de Autodeterminación menor que el 56.8\% de la población con DI.

- Presenta un nivel de Inclusión Social menor que el $75.1 \%$ de las personas con DI.

- Se sitúa por debajo del 95.1\% de la población con DI en el índice de Derechos.

El parámetro que alcanza las puntuaciones más altas es el de desarrollo personal (43.5), seguido por el de autodeterminación (43.1) y bienestar emocional (27.3). Bienestar físico (2.1) y derechos (4.9) obtienen puntuaciones muy bajas.

Comparando los índices de CV con la edad de los participantes, los resultados muestran que no existen diferencias estadísticamente significativas 
entre ambas variables. Observamos diferencias estadísticamente significativas únicamente en el grado de desarrollo personal entre los participantes de 21-34 años y los de 45-65 ( $\mathrm{F}=3.9$; $\mathrm{p}>.05)$. Los participantes más jóvenes muestran un nivel superior de desarrollo personal (media = 10.8) que los mayores de 45 años (media $=9.1$ ), siendo estos los que poseen las puntuaciones más bajas de los tres grupos de edad.

Aunque no se observan diferencias estadísticamente significativas en el resto de las subescalas, el análisis de las puntuaciones muestra algunas tendencias en el perfil de CV en función del grupo de edad, tal y como puede observarse en la figura 2 .

Figura 2

Puntuaciones medias en las subescalas de CV por edades

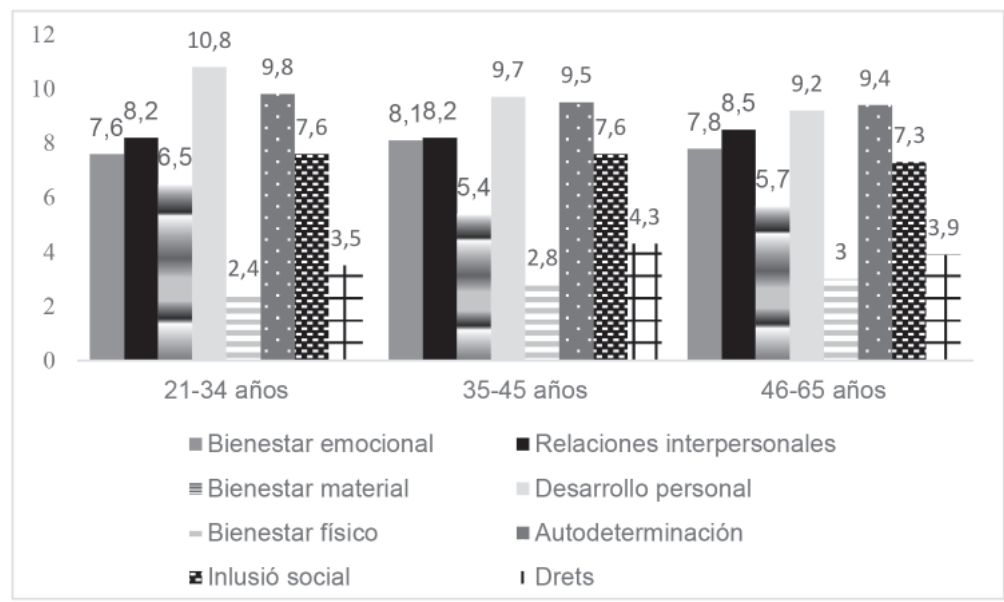

El grupo de 21 a 34 años obtiene puntuaciones más altas en desarrollo personal, bienestar material y autodeterminación. Por el contrario, son el grupo más bajo en cuanto a bienestar físico. El grupo de edad avanzado (de 46 a 65 años), obtiene las puntuaciones más altas en relaciones interpersonales y en bienestar físico. Tanto el grupo de "jóvenes" como el de "mediana edad" obtienen el mismo nivel de puntuaciones en las variables inclusión social y relaciones interpersonales.

Comparando los índices de CV con el género de los participantes, los resultados muestran que no existen diferencias estadísticamente significativas entre hombres y mujeres; la puntuación estándar compuesta media oscila entre 56.2 (hombres) y el 54 (mujeres).

Si analizamos las puntuaciones obtenidas por ambos grupos a partir de la interpretación del test, observamos que los hombres han obtenido una 
puntuación superior solo al 9.5\% de la población y las mujeres al 7.6\%. Ello indica una mejor CV en los hombres, sin embargo, también indica que más del $85.5 \%$ de la población general obtiene puntuaciones superiores a los integrantes de esta muestra, independientemente del género.

En relación con el perfil de CV, se observan diferencias estadísticamente significativas entre género en las relaciones interpersonales $(t=3.133 \mathrm{p}>$ $.05)$, observando puntuaciones mayores entre los hombres (media $=9.1)$ que entre las mujeres (media $=7.8$ ).

Aunque no se observan diferencias estadísticamente significativas en el resto de las subescalas, el análisis de las puntuaciones muestra un perfil de CV diferenciado en función del género, tal y como puede observarse en la figura 3.

Los hombres obtienen mayores puntuaciones en 4 dimensiones (bienestar emocional, relaciones interpersonales, inclusión social y derechos), mientras que las mujeres solo en 2 (bienestar físico y bienestar material). En el parámetro de autodeterminación, ambos grupos obtienen casi la misma puntuación (9.5 hombres y 9.4 mujeres).

Figura 3

Puntuaciones medias en las subescalas de CV por género

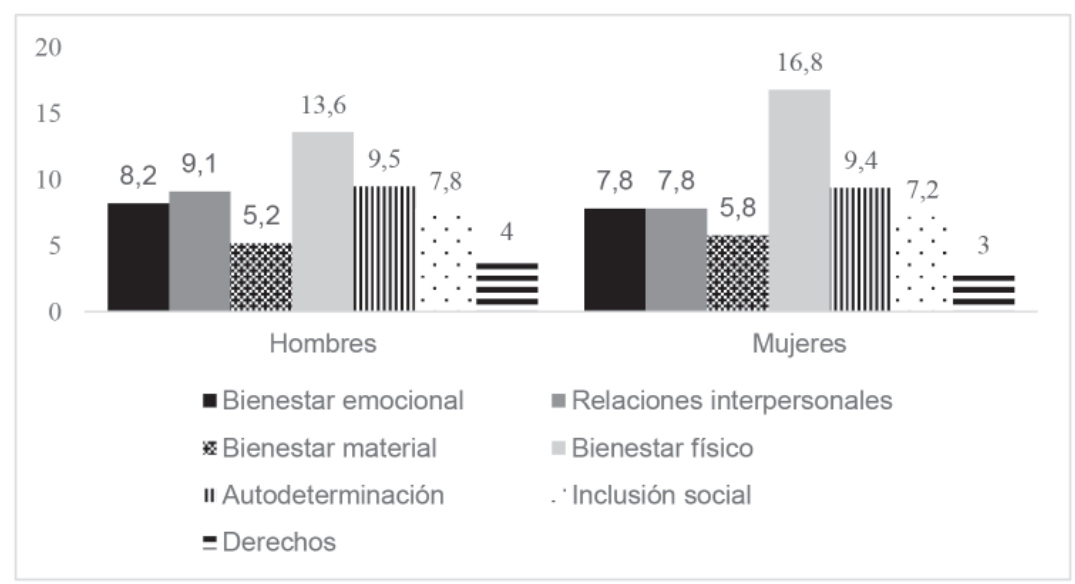

\section{Competencias de empleabilidad}

Los resultados generales demuestran que el desarrollo de las 4 competencias analizadas es muy similar. Las puntuaciones medias se sitúan entre el 3.2 (para las cognitivas y de aprendizaje) y 3.1 para las restantes, por lo que se puede afirmar que, en general, tienen un buen nivel de 
competencias, tomando en consideración que se evaluan con una escala de 1 a 4 (ver figura 4 ).

Figura 4

Niveles de competencia por subescala

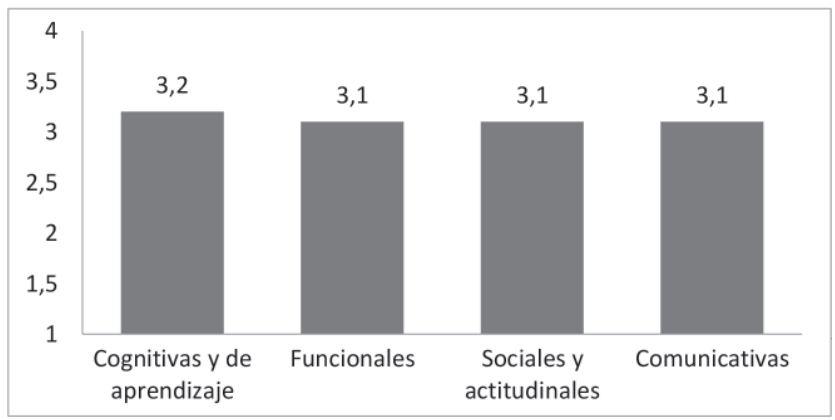

No existe mucha dispersión entre las puntuaciones de las competencias cognitivas y de aprendizaje ( $\mathrm{DS}=.69)$, las funcionales $(\mathrm{DS}=.52)$ y las sociales-actitudinales ( $\mathrm{DS}=.48$ ) en las que la DS es menor a 1 en los tres casos, mientras que se observa una mayor dispersión en las competencias comunicativas (DS=.75) en la que la desviación estándar se acerca a 1.

Comparando el nivel de competencias con la edad de los participantes, los resultados muestran que no existen diferencias estadísticamente significativas entre los diferentes grupos de edad y el nivel general de competencias. Tampoco se observan diferencias en los niveles de las diferentes subescalas. Sin embargo, sí que se observan algunas tendencias tal y como muestra la figura 5 .

Figura 5

Puntuaciones medias en las subescalas de competencias por edad

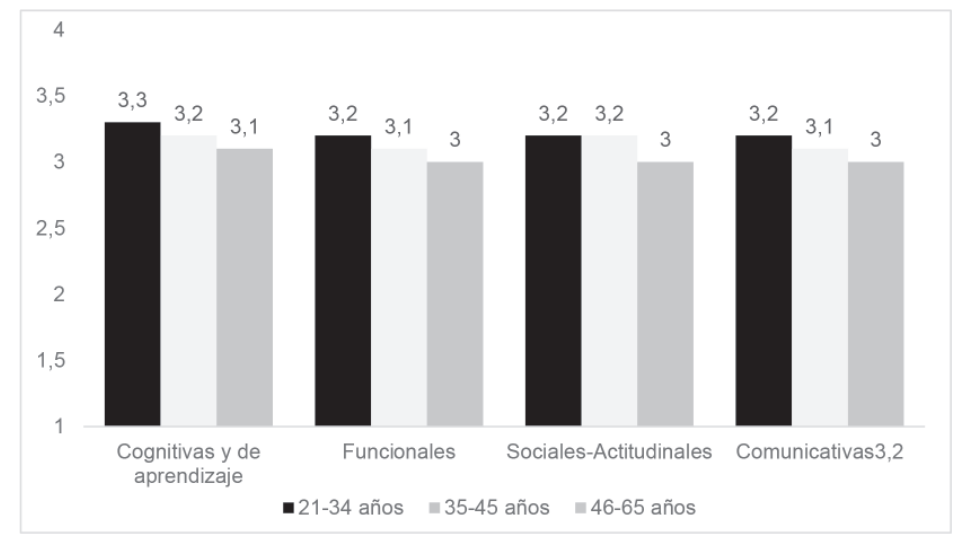


En una escala del 1 al 4 todas las subescalas se sitúan en una puntuación media de 3, con lo que se puede afirmar que el nivel competencial es óptimo.

Comparando el nivel de competencias de los participantes teniendo en cuenta el género, los resultados muestran que no existen diferencias estadísticamente significativas entre hombres y mujeres en el nivel de competencias general, ni tampoco en las subescalas. Se muestra un perfil similar en función del género, donde se observa un nivel alto en el desarrollo de cada una de las competencias (nivel medio de 3 en una escala de 1 a 4), tal y como puede observarse en la figura 6 .

Figura 6

Puntuaciones medias de la escala de competencias de empleabilidad en función del género

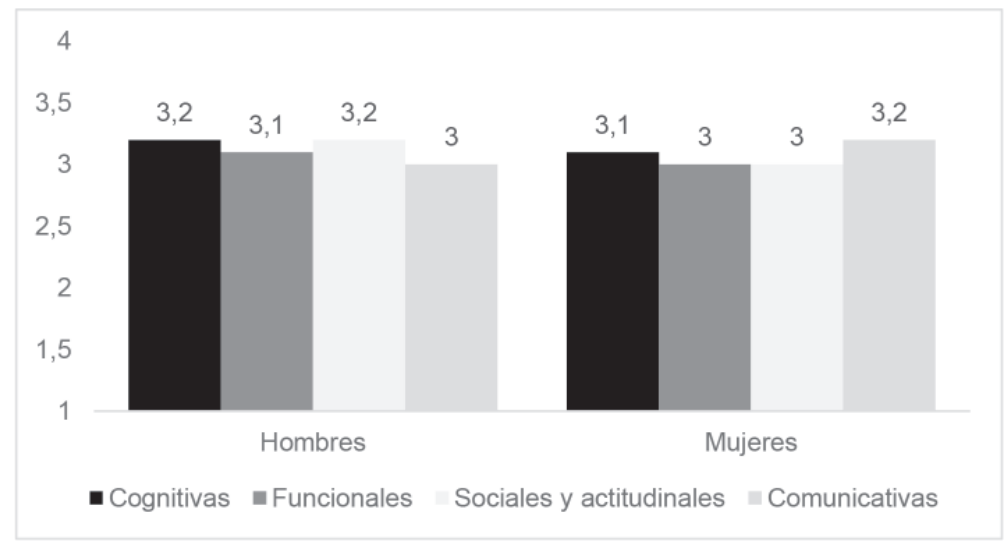

\section{Relación entre calidad de vida y competencias de empleabilidad}

No existe relación entre el índice de CV y el nivel de competencias general, ni entre el nivel de competencia general y las subescalas de CV.

Analizando las diferentes escalas se observa una relación moderada estadísticamente significativa entre las competencias sociales y actitudinales y el bienestar emocional $(\mathrm{r}=.219 ; \mathrm{p}>.05)$ y las relaciones interpersonales $(\mathrm{r}=$ $.260 ; \mathrm{p}>.05)$.

\section{CONCLUSIONES Y DISCUSIÓN}

Los resultados muestran que las personas trabajadoras de los CEE tienen un bajo índice de CV. En general, la muestra analizada obtiene puntuaciones inferiores en $\mathrm{CV}$ respecto al colectivo de referencia, sobre todo 
en bienestar físico y derechos. Los resultados coincidentes con el estudio de Badía et al. (2016), invitan a diseñar intervenciones que mejoren estas dos dimensiones, así como a elaborar planes individualizados. En este sentido, coincidimos con Muntaner (2013) al considerar la necesidad de planificar acciones educativas centradas en la persona para mejorar su índice de CV. Quizás el sometimiento al estrés laboral, tal y como apuntan Flores et al. (2010) sea un factor que afecta la CV en el trabajo de estas personas.

El análisis de las ocho dimensiones de CV revela bajas puntuaciones en todas ellas, siendo las más altas "desarrollo personal" y "autodeterminación" y la más baja "bienestar físico". Los trabajos de Badía et al. (2016) y Rodríguez y Tortosa (2016) coinciden con los resultados obtenidos, excepto en la dimensión de bienestar físico, al obtener en ambas investigaciones resultados altos. Cabe considerar que mientras que Castro et al. (2016) obtuvieron bajas puntuaciones en autodeterminación, en el presente estudio ha sido una de las dimensiones con valoraciones más altas.

El análisis del desarrollo de las competencias de empleabilidad muestra niveles altos, esto nos conduce a pensar que el proceso de supervisión y apoyo en el puesto de trabajo realizado por los CEE potencia este tipo de competencias. Para O'Reilly (2007), el alto nivel competencial de estas personas es debido al encaje entre puestos de trabajo y competencias, intereses y aptitudes. Lo cierto es que si tal y como afirman Dalmau et al. (2013) una de las limitaciones de las personas con DI para acceder al mercado de trabajo ordinario es la falta de competencias de empleabilidad, no es el caso de estos trabajadores con lo que estaríamos de acuerdo con Peña y Santana-Vega (2020) al afirmar que es necesario prepararlos para esta transición puesto que demuestran la adquisición de estas competencias.

La edad y el género no influyen directamente en el índice general de $\mathrm{CV}$ en los trabajadores de los CEE. Sin embargo los jóvenes demuestran mayores niveles de desarrollo personal que los adultos de edad avanzada. También se perciben mejores puntuaciones en desarrollo personal, bienestar material y autodeterminación en los trabajadores jóvenes, mientras que los adultos de edad avanzada, en relaciones interpersonales y bienestar físico. Sin embargo, sí existe significatividad en alguna de las subescalas de la CV. Los hombres han obtenido mayor puntuación en las relaciones interpersonales que las mujeres. Y se percibe un nivel más alto en bienestar emocional, inclusión social y derechos en los hombres, mientras que las mujeres en bienestar físico y material.

Tal y como afirman Alcedo et al. (2017), existen pocos estudios sobre la variable género y la $\mathrm{CV}$ en este colectivo, por lo que deberían realizarse 
estudios profundos para localizar las necesidades de las persones con DI en función de esta variable, y poder generar soluciones socioeducativas.

En cuanto a la edad, por ejemplo, las relaciones estadísticamente significativas entre los jóvenes y el grado de desarrollo personal y el de autodeterminación, implican la necesidad de profundizar en esta línea de trabajo en estadios vitales posteriores, y no solo centrarlo en la juventud. Los mayores de 45 años muestran niveles inferiores en todas las dimensiones de $\mathrm{CV}$, evidenciándose la necesidad de planificar actuaciones educativas que mejoren la $\mathrm{CV}$ de los mayores con DI, lo que también requiere un trabajo preventivo para favorecer una vida plena y satisfactoria a lo largo de su ciclo vital. Las investigaciones de Hole et al. (2013) y las de Lehmann et al. (2013) consideran necesario que las acciones educativas se centren en potenciar la inclusión en la comunidad, la autodeterminación y el apoyo familiar para promover un envejecimiento activo de calidad.

Además, existen otros factores a tener en cuenta, como la consideración del inicio de la tercera edad en estadios tempranos, o las especificidades que presenta el colectivo en dicha etapa vital (Alcedo et al., 2017; o Cruz Díaz y Jiménez Gómez, 2017). Sería necesario investigar sobre si la anticipación de la etapa de la vejez aplica también a las personas con DI leve.

Tampoco influye la edad y el género en el grado de desarrollo de las competencias de empleabilidad, aunque se percibe un nivel más bajo en los trabajadores de edad avanzada. En cuanto al género, el hecho de que los hombres obtengan un índice mayor, y estén mejor posicionados en 4 subescalas de competencias, mientras que las mujeres solo en dos, apunta la necesidad de un trabajo específico con el colectivo de mujeres con discapacidad, por sufrir una doble discriminación (Flores et al. (2015).

Otro de los hallazgos del estudio es la inexistencia de una relación estadísticamente significativa entre el nivel competencial general y el índice de CV. Este dato es sorprendente, puesto que las competencias de empleabilidad analizadas son transferibles a otros ámbitos de la vida de la persona y no solo el laboral. Estos datos apuntan hacia una relación no directamente proporcional entre ambas variables, es decir, que los participantes de la investigación posean altas competencias de empleabilidad no ha influido en su nivel de CV y viceversa. Estos resultados no coinciden con Arellano y Peralta (2013) puesto que en su investigación encontraron relación entre ambas variables, aunque cabe destacar que ellos utilizaron como informantes los padres de las personas con DI, mientras que en el presente estudio han sido los supervisores laborales (evaluación objetiva). Estudios, como el de Alomar y Cabré (2005), revelaron que la inserción laboral influye en el desarrollo de la CV. Deberíamos profundizar en este 
hallazgo, puesto que parece razonable inferir que las personas con altos niveles de competencias cognitivas y de aprendizaje, funcionales, socialesactitudinales y comunicativas deberían manifestar también altos niveles de $\mathrm{CV}$. Cabe tener en cuenta que el nivel de CV de estos trabajadores está por debajo de la media de la población; quizás este hecho ha provocado este resultado inesperado.

Los bajos niveles de CV que demuestran los participantes en esta investigación nos conducen a afirmar la necesidad de emprender acciones para garantizar una buena CV de las personas con DI. Coincidimos con Schalock y Verdugo (2007) y Muntaner (2013), en que la mejora de la $\mathrm{CV}$ requiere acciones longitudinales que se inicien en los primeros años de vida, como por ejemplo cambios en la escuela para que sea realmente inclusiva. En este sentido, Durán y Giné (2011) consideran que la educación inclusiva está íntimamente ligada al desarrollo integral de la persona, y que las propuestas educativas deben adaptarse a la diversidad del alumnado y promover más y mejores oportunidades para todos.

Para finalizar, otro factor que apunta hacia la necesidad de entornos inclusivos es la evidencia de la influencia positiva de normalización en la $\mathrm{CV}$ de personas con discapacidad. Así, el estudio desarrollado por Muñoz et al. (2016), por ejemplo, demuestra que los jóvenes de hasta 19 años, escolarizados en centros de educación especial, presentan menores índices de $\mathrm{CV}$ en parámetros como el bienestar físico o la autodeterminación (que incluye factores tan relevantes como el nivel de independencia y/o participación en la toma de decisiones sobre cómo pasar el tiempo libre, decidir sobre la propia vida personal, gastar el dinero, con quién vivir y, en definitiva, como organizar su propia vida), que los escolarizados en régimen ordinario. 


\section{REFERENCIAS BIBLIOGRÁFICAS}

Alcedo, M. A, Fontanil, Y., Solís, P., Pedrosa, I., \& Aguado, A. L. (2017). People with intelectual disability who are ageing: preceived needs assessment. Intenational Journal of Clinical and Health Psychology 17, 38-45. https://doi.org/10.1016/j. ijchp.2016.07.002

Alomar, E. y Cabré, M. (2005). El trabajo de jóvenes con discapacidad intelectual en entornos normalizados. Revista Síndrome de Down, 22, 118124. https://bit.ly/2MMVVy4

Arellano, A. y Peralta, F. (2013). Calidad de vida y autodeterminación en personas con discapacidad. Valoraciones de los padres. Revista Iberoamericana de Educación, 63. https://doi.org/10.35362/rie630561

Badía, M., Carrasco, J., Orgaz, M.B., y Escalonilla, J.M. (2016). Calidad de vida percibida por personas adultas con discapacidades del desarrollo versus la informada por profesionales. Siglo Cero, 47(1), 7-21. http://dx.doi. org/10.14201/scero20161721

Blanco, A. (2008). Formación universitaria basada en competencias. En L. Prieto (Ed.), La enseñanza universitaria centrada en el aprendizaje (pp. 31-59). Octaedro.

Cruz Díaz, M.R. y Jiménez Gómez, M.V. (2017). Envejecimiento y discapacidad intelectual. Aproximación a las necesidades de las personas adultas y mayores con discapacidad intelectual y sus familias. International Journal of Educational Research and Innovation 7, 76-90. https://bit.ly/2XSTYXn

De Cuyper, N., Mauno, S., Kinnunen, U., \& Makikangas, A. (2011). The role of job resources in the relation between perceived employability and turnover intention: A prospective twosample study. Journal of Vocational Behavior, 78 (2), 253-263. https://doi. org/10.1016/j.jvb.2010.09.008

De la Fuente Anuncibay, R. y GonzálezCastro, J. (2009). Empleo, formación e inserción de colectivos en riesgo de exclusión. Un reto social y económico para la empresa receptora. Bordón, 61(3), 33-45. https://bit.ly/3h6TAfc

De Jesús, A., Girón, I., y Garcia, G. (2009). Relación entre la modalidad de empleo y el nivel de calidad de vida laboral de personas con discapacidad intelectual leve. Nómadas. Revista Crítica de Ciencias Sociales y Jurídicas, 22(2), 1-50. https://bit.ly/3hc7n4c

Docampo, G. y Morán, M.C. (2014). Evaluación de la competencia profesional en personas con discapacidad intelectual. Una propuesta de adaptación de la metodología e instrumentos INCUAL para la igualdad de oportunidades. Revista Española de Discapacidad, 2(1), 71-96. https://doi.org/10.5569/23405104.02.01.04

Durán Gisbert, D. y Giné Giné, C. (2011). La formación del profesorado para la educación inclusiva: Un proceso de desarrollo profesional y de mejora de los centros para atender la diversidad. Revista Latinoamericana de Educación Inclusiva, 5(2), 150-173. https://bit. ly/2XNJsQN

Ellenkamp, J.J.H., Brouwers, E.P.M., Embregts, P.J.C. M., Joosen, M.C.W., \& van Weeghel, J. (2016). Work environment-related factors in obtaining and maintaining work in a competitive employment setting for employees with intellectual disability: A systematic review. Journal of 
Occupational Rehabilitation, 26, 5669. https://doi.org/10.1007/s10926-0159586-1

Fillary, R. \& Pernice, R. (2005). Workplace culture analysis where people with intellectual disabilities work: A case study approach. Journal of Intellectual and Developmental Disability, 30(3), 176-180. https://doi. org/10.1080/13668250500204091

Flores, N., Jenaro, C., Martinelli, P., y Vega, V. (2015). Desigualdad de género, inclusión laboral y riesgos psicosociales: evidencias en trabajadoras con discapacidad intelectual. Cuestiones de género: de la igualdad y la diferencia 9, 89-114. https://doi.org/10.18002/cg.v0i9.1153

González, H. y Laborda, C. (2017). Usuarios con discapacidad intelectual leve y moderada en centros ocupacionales: competencias laborales y calidad de vida. En A. RodríguezMartín (Ed.), Prácticas innovadoras inclusivas: retos y oportunidades (pp. 2647-2654). Servicio de Publicaciones Universidad de Oviedo

Gilson, C., Carter, E., \& Biggs, E. (2017). Systematic Review of Instructional Methods to Teach Employment Skills to Secondary Students with Intellectual and Developmental Disabilities. Research and Practice for Persons with Severe Disabilities 42(2), 89-107. https://doi. org/10.1177/1540796917698831

Grup de Treball d'Orientació (2010). La transició a la vida activa de l'alumnat amb barreres per a l'aprenentatge $i$ la participación. Consorci d'educació de Barcelona. https://bit.ly/3hebZa4

Hardoon, D. (2017). Informe Oxfam: Una economía para el 99\%. https://bit. ly/3fe66rp
Hole, R.D., Stainton, T., \& Wilson, L. (2013). Ageing Adults with Intellectual Disabilities: Self-advocates' and Family Members' Perspectives about the Future. Australian Social Work, 66, 571-589. https://doi.org/10.1080/03124 07X.2012.689307

Holmes, J. \& Fillary, R. (2000). Handling Small Talk at Work: challenges for workers with intellectual disabilities. International Journal of Disability, Development and Education, 47(3), 273291. https://doi.org/10.1080/713671114

Jahoda, A., Kemp, J., Riddell, S., \& Banks, P. (2008). Feelings about work: A review of the socio-emotional impact of supported employment on people with intellectual disabilities. Journal of Applied Research in Intellectual Disabilities, 21(1), 1-18. https://doi. org/10.1111/j.1468-3148.2007.00365.x

Laborda, C. y González, H. (2017a). Características diferenciales del empleo de personas con discapacidad intelectual. En A. Rodríguez-Martín (Ed.), Prácticas innovadoras inclusivas: retos y oportunidades (pp. 2621-2628), Servicio de Publicaciones Universidad de Oviedo.

Laborda Molla, C. y González Fernández, H. (2017b). Estudio cualitativo sobre discapacidad intelectual y trabajo protegido. La orientación como herramienta para mejorar la calidad de vida. Revista Española de Orientación y Psicopedagogía 28 (3), 82-98. https://doi.org/10.5944/reop. vol.28.num.3.2017.21620

Lehmann, B.A., Bos, A.E.R., Rijken, M., Cardol, M., Peters, G.J.Y., Kok, G., \& Curfs, L.M.G. (2013). Ageing with an intellectual disability: the impact of personal resources on wellbeing. Journal of Intellectual Disability 
Research, 57, 1068-1078. https://doi. org/10.1111/ j.1365-2788.2012.01607

Lindstrom, L., Doren, B., \& Miesch, J. (2011). Waging a Living: Career development and long-term employment outcomes for young adults with disabilities. Exceptional Children, 77(4), 423-434. https://doi. org/ 10.1177/001440291107700403

Lucas, S; Arias, B., y Ovejero A. (2005). Orientación profesional e inserción sociolaboral de personas con discapacidad intelectual. Revista universitaria de ciencias del trabajo, 6, 393-414. https://bit.ly/3fe66rp

Moratalla Santamaría, P. (2016). Centros Especiales de Empleo. Revista jurídica de economía social y cooperativa, 29, 235-274

Moratalla, P. (2016). Centros especiales de empleo. CIRIEC-España Revista Jurídica 29, 1-38. https://bit.ly/2XLSLki

Muñoz Cantero, J., Losada Puente, L., \& Silva Almeida, L. (2016). Quality of life, adolescence and inclusive schools: comparing regular and special needs students. Bordón. Revista de Pedagogía, 69(1), 139-154. https://doi. org/10.13042/Bordon.2016.48977

Muntaner Guasp, J.J. (2013). Calidad de vida en la escuela inclusiva. Revista Iberoamericana de Educación, 63, 3549. https://bit.ly/2MXLlo9

Nota, L., Ginevra, M.C., \& Carrieri, L. (2010). Career interests and selfefficacy beliefs among young adults with an intellectual disability. Journal of Policy and Practice in Intellectual Disabilities, 7(4), 250-260. https://doi. org/10.1111/j.1741-1130.2010.00274.x

O’Reilly, A. (2007). El derecho al trabajo decente de las personas con discapacidades. Oficina Internacional del Trabajo. https://bit.ly/3cJVxuI
Pegalajar Palomino, M.C. y Xandri Martínez, R. (2015). La inclusión sociolaboral de jóvenes con discapacidad intelectual: una experiencia a partir del programa "Capacitas". Revista nacional e internacional de educación inclusiva, 8(1), 59-73. https://bit.ly/2zkzCwt

Peña, M.T. y Santana-Vega, L.E. (2020). Transición al empleo de personas con discapacidad intelectual en Canarias: el empleo con apoyo. MLS Educational Research, 4(1),1-16. https://doi. org/10.29314/mlser.v4i1.321

Real Decreto 2273/1985, de 4 de diciembre, por el que se aprueba el Reglamento de los Centros Especiales de Empleo definidos en el artículo 42 de la Ley 13/1982, de 7 de abril, de Integración Social del Minusválido. https://bit.ly/2UwgOBN

Rodríguez González, N. (2019). Inserción laboral de personas con discapacidad y medidas establecidas para su fomento. https://bit.ly/2zjwD7w

Rodríguez Gregory, A. y Tortosa, J. (2016). Nivel de condición física y calidad de vida en personas con discapacidad intelectual. Actividad Física y Deporte: Ciencia y Profesión, 24, 13-28. https://bit.ly/3cOh71k

Romeo, M., Yepes-Baldó, M., Pascual, C., Barbancho, F., Posada, D.M., y Boria, S. (2017). Proyecto DIL-D CEE: Desarrollo de políticas y estrategias de integración laboral de personas con Discapacidad en Centros Especiales de Empleo. Càtedra per a la integració de persones amb discapacitat. Universitat de Barcelona. Fundació Addeco. https://bit.ly/3f98Pm1

Stephens, D.L., Collins, M.D., \& Dodder, R.A. (2005). A longitudinal study of employment and skill acquisition among individuals with developmental 
disabilities. Research in Developmental Disabilities, 2(5), 469-486. https://doi. org/10.1016/j.ridd.2003.12.003.

Verdugo, M. A., Arias, B., Gómez, L.E., y Schalock, R.L. (2008). Formulari de
l'Escala Gencat de Qualitat de vida. Manual d'aplicació de l'Escala Gencat de Qualitat de vida. Departament de Acció Social i Ciudadania, Generalitat de Catalunya. https://bit.ly/2MMR4wU 


\section{PERFIL ACADÉMICO Y PROFESIONAL DE LAS AUTORAS}

Cristina Laborda Molla. ORCID: http://orcid.org/0000-0002-8709-6978

Profesora Titular de Atención a la Diversidad del área de Didáctica y Organización Educativa del Dpto. de Pedagogía Aplicada de la Universitat Autònoma de Barcelona. Líneas de investigación: inclusión sociocomunitaria, ocio, inserción laboral, calidad de vida y promoción de vida autónoma de personas con discapacidad intelectual. Actualmente desarrolla un estudio sobre el impacto de un nuevo modelo ocupacional inclusivo y territorial de personas con DI. Investigadora del grupo Diversidad y Orientación (DOUAB). E-mail: cristina.laborda@uab.cat

Mercè Jariot García. ORCID: http://orcid.org/0000-0002-5061-2940

Profesora Agregada de Orientación Profesional del área de Métodos de Investigación y Diagnóstico en Educación, del Dpto. de Pedagogía Aplicada de la Universitat Autònoma de Barcelona.

Líneas de investigación: orientación profesional, formación y educación vial, intervención educativa asistida con animales, procesos de orientación en la infancia y la adolescencia en riesgo. Investigadora principal del grupo de investigación ERESv (Equip de Recerca en Educació i Seguretat viàries). E-mail: merce.jariot@uab.cat 7318

Henar González Fernández. ORCID: http://orcid.org/0000-0002-4520-

Profesora Asociada del área de de Didáctica y Organización Educativa del Dpto. de Pedagogía Aplicada de la Universitat Autònoma de Barcelona.

Líneas de investigación: comunicación en menores con sordoceguera, orientación sociolaboral, calidad de vida y competencias laborales en personas con discapacidad intelectual. Actualmente investiga el desarrollo profesional de personas con DI. Es investigadora del grupo Diversidad y Orientación (DO-UAB). E-mail: henar.gonzalez@uab.cat

Fecha Recepción del Artículo: 27. Enero. 2020

Fecha Modificación del Artículo: 10. Junio. 2020

Fecha Aceptación del Artículo: 18. Junio. 2020

Fecha Revisión para Publicación: 13. Julio. 2020 
